\title{
AN OVERLAPPING ADDITIVE SCHWARZ-RICHARDSON METHOD FOR MONOTONE NONLINEAR PARABOLIC PROBLEMS*
}

\author{
M. MUNTEANU ${ }^{\dagger}$ AND L. F. PAVARINO!
}

\begin{abstract}
We construct and study a scalable overlapping Additive Schwarz-Richardson (ASR) algorithm for monotone nonlinear parabolic problems discretized implicitly in time. At each time step, the Additive Schwarz preconditioner is built using the linear part of the nonlinear operator, partitioning the domain of the problem into overlapping subdomains, solving local problems on these subdomains and solving an additional coarse problem associated with the subdomain mesh. This preconditioner is then applied to the nonlinear operator using a Richardson iteration. We prove first an abstract convergence result and then convergence rate estimates showing the scalability of the ASR algorithm. The results of numerical experiments in the plane confirm the theoretical estimates and illustrate the performance of the one and two-level ASR algorithm and in the presence of discontinuous coefficients in the parabolic operator.
\end{abstract}

Key words. monotone nonlinear parabolic problems, domain decomposition preconditioners, overlapping additive Schwarz, finite elements, implicit time discretizations

AMS subject classifications. 65M55, 65H05

1. Introduction. In recent years, domain decomposition methods have been extended in different ways to nonlinear problems arising in many application areas. As a first approach, domain decomposition methods provide preconditioners for the Jacobian system in a Newton-like iteration. In this context, Schwarz-type preconditioners have been successfully used by Cai et al. to solve problems from various applied fields, e.g., computational fluid dynamics [11, 17], full potential problems [10], cardiac electrical activity [25], and unsteady nonlinear radiation diffusion [27]. Additive Schwarz methods have been used not only as inner iterations in a Newton-Krylov-Schwarz (NKS) scheme, but also as outer iterations in nested solvers such as ASPIN $[12,1]$ or in the nonlinear additive Schwarz method by Dryja and Hackbusch [15]. Different approaches have been studied by Lui [19, 20] using the method of monotone iterations for continuous nonlinear elliptic and parabolic PDEs and by Boglaev [4] using monotone Schwarz methods at the discrete level for singularly perturbed reaction-diffusion problems. Extensions of the classical additive and multiplicative Schwarz methods have been studied by Tai and Espedal [31,32] for convex programming problems and by Badea [2] for constrained minimization problems.

In this paper, we follow instead the work of Cai and Dryja [9] for monotone elliptic problems and extend it to monotone nonlinear parabolic problems. The main idea is to build an overlapping additive Schwarz preconditioner for the linear part of the operator, partitioning the domain of the problem into overlapping subdomains, solving local problems on these subdomains, and solving an additional coarse problem associated with the subdomain mesh. This preconditioner is then applied to the nonlinear operator using a Richardson iteration. The linearity of the preconditioner allows us to employ the main technical tools of the classical abstract theory of additive Schwarz methods (see, e.g., [33]), and prove an abstract convergence result for the resulting iterative method. With this result, we can then obtain precise convergence estimates for the Additive Schwarz-Richardson (ASR) method applied to the time discretization of monotone nonlinear parabolic problems. The two-level ASR method turns out to be scalable and with a convergence rate depending only on the ratio $H / \delta$ as in the

* Received December 19, 2007. Accepted for publication September 1, 2008. Published online on December 17, 2008. Recommended by T. Manteuffel. This work was supported by Istituto Nazionale di Alta Matematica Francesco Severi, Roma and by M.I.U.R. (PRIN 2004014411, 2005013982).

${ }^{\dagger}$ Department of Mathematics, Università di Milano, Via Saldini 50, 20133 Milan, Italy (\{Marilena.Munteanu, Luca.Pavarino\}@mat.unimi.it). 
linear case, where $H$ is the subdomain characteristic size and $\delta$ the overlap size. Without a coarse space, the one-level ASR method can still have a constant upper bound if the time step size $\tau$ is small enough. Otherwise, the convergence rate depends on the ratio $\tau /(H \delta)$ and scalability is lost as in the linear case. In case of generous overlap $\delta=C H$, these estimates agree with the estimates obtained by Cai $[7,8]$ for linear parabolic problems.

The rest of the paper is organized as follows. In Section 2, we introduce the nonlinear parabolic problem, its main properties and time discretization. In Section 3, we define the ASR method in both its functional and matrix form. An abstract convergence result is given in Section 4, where we prove some technical lemmas leading to the main result of Theorem 4.7. In Section 5, this abstract result is applied to the time discretization of our nonlinear parabolic problem and convergence rate estimates are obtained for both one- and two-level ASR methods. Section 6 concludes the paper with the results of several numerical experiments in the plane, confirming the theoretical results obtained and illustrating the scalability of the ASR method. We also compare the ASR method with the Linearly Implicit Euler method, based on solving an appropriate linear system involving the Jacobian of the nonlinear operator by using GMRES with the Additive Schwarz preconditioner as in ASR and we show that the ASR method is asymptotically less expensive.

2. Continuous and discrete nonlinear parabolic problems. We consider a polyhedral domain $\Omega \subset R^{d}, d=2,3$, with Lipschitz continuous boundary $\partial \Omega$, the spaces

$$
V=\left\{v \in H^{1}(\Omega): v=0 \text { on } \Gamma_{1} \subset \partial \Omega, \operatorname{meas}\left(\Gamma_{1}\right)>0\right\}, L^{2}(\Omega),
$$

and the nonlinear form $b: H^{1}(\Omega) \times H^{1}(\Omega) \longrightarrow \mathbf{R}$ satisfying the following properties:

1. $b$ is Lipschitz continuous: $\exists L>0$ such that, $\forall v, w, z \in H^{1}(\Omega)$, $|b(v, z)-b(w, z)| \leq L\|v-w\|_{H^{1}(\Omega)} \cdot\|z\|_{H^{1}(\Omega)}$;

2. $b$ is bounded: $\exists C>0$ such that $|b(v, w)| \leq C\left(1+\|v\|_{H^{1}(\Omega)}\right)\|w\|_{H^{1}(\Omega)}$, $\forall v, w \in H^{1}(\Omega)$;

3. $b$ is hemicontinuous: $\forall u, v, w \in H^{1}(\Omega)$, the function $\alpha \longrightarrow b(u+\alpha v, w)$ is continuous;

4. $b$ is strictly monotone: $b(v, v-w)-b(w, v-w) \geq 0, \forall v, w \in H^{1}(\Omega)$, and equality holds only for $v=w$;

5. $b$ is linear in its second argument: $b\left(v, \sum_{i=1}^{n} \alpha_{i} w_{i}\right)=\sum_{i=1}^{n} \alpha_{i} b\left(v, w_{i}\right), \forall v, w_{i} \in H^{1}(\Omega)$, $\forall \alpha_{i} \in \mathbf{R}, i=1, \ldots, n$

6. $b(v, v) \geq c\|v\|_{H^{1}(\Omega)}^{2}-c_{0}\|v\|_{H^{1}(\Omega)}-c_{1}\|v\|_{L^{2}(\Omega)}^{2}-c_{2}, \forall v \in H^{1}(\Omega)$, where $c>0, c_{0}>0, c_{1} \geq 0, c_{2} \geq 0$ are constants.

We consider the following nonlinear parabolic problem: given $u_{0} \in L^{2}(\Omega)$ and $f \in L^{2}\left((0, T) ; V^{*}\right)$, find $u \in W \equiv\left\{u \in L^{2}((0, T) ; V), u^{\prime} \in L^{2}\left((0, T) ; V^{*}\right)\right\}$, such that

$$
\left\{\begin{array}{l}
<u^{\prime}(t), w>+b(u(t), w)=<f(t), v>, \forall t \in(0, T) \backslash E_{w}, \forall w \in V, \\
u(0)=u_{0},
\end{array}\right.
$$

where $E_{w} \subset(0, T)$ is a set of measure zero that depends on the function $w$.

The continuous problem (2.1) is discretized implicitly in time by the backward Euler method and in space by the finite element method. We suppose for simplicity that the time interval $(0, T)$ is discretized with a uniform time step $\tau=T / M$ into $M$ equal subintervals and that the domain $\Omega$ is discretized with a regular finite element triangulation $\mathcal{T}_{h}$ with mesh size $h$. The associated piecewise linear finite element space $V_{h}$ is defined by

$$
V_{h}=\left\{v \mid v \text { is continuous on } \bar{\Omega},\left.v\right|_{k} \text { is linear } \forall k \in \mathcal{T}_{h}, v=0 \text { on } \bar{\Gamma}_{1}\right\} \text {. }
$$


We denote by $u_{h}^{m} \in V_{h}$ the finite element approximation of a function $u \in V$ at time $t^{m}=m \tau$ and let $f^{m}=\frac{1}{\tau} \int_{t_{m-1}}^{t_{m}} f(t) d t$. We then obtain the following fully discrete problem: given an arbitrary sequence $\left\{u_{h}^{0}\right\} \subset L^{2}(\Omega)$ of approximations of $u^{0}$, such that $\lim _{h \rightarrow 0}\left\|u_{h}^{0}-u^{0}\right\|=0$, find $u_{h}^{m} \in V_{h}$, such that

$$
\left(\frac{u_{h}^{m}-u_{h}^{m-1}}{\tau}, v\right)+b\left(u_{h}^{m}, v\right)=<f^{m}, v>, \forall v \in V_{h} .
$$

Results on the existence and uniqueness of the solution of the discrete and continuous parabolic problems can be found, e.g., in [35, Theorems 45.3 and 46.4], respectively. The convergence of the discrete solution to the continuous one is presented in [35, Theorems 46.4 and 47.1].

3. An Additive Schwarz-Richardson algorithm. Since in the rest of the paper we only consider discrete functions, for simplicity, we drop the indices $h$ and $m$ and denote by $u$ both the finite element approximation $u=\sum_{j=1}^{n} u_{j} \phi_{j}$ of the continuous solution in the finite element basis $\left\{\phi_{j}, j=1, \ldots, n\right\}$ of $V_{h}$, and its vector representation $u=\left[u_{1}, \ldots, u_{n}\right]^{T}$ Problem (2.2) can then be written as the nonlinear algebraic system,

$$
B(u)=\hat{g},
$$

where

$$
\begin{array}{lll}
B(u)=\left[B_{1}, \ldots, B_{n}\right]^{T}, & \text { with } & B_{j}=\left(u, \phi_{j}\right)+\tau b\left(u, \phi_{j}\right), \\
\hat{g}=\left[g_{1}, \ldots, g_{n}\right]^{T}, & \text { with } & g_{j}=\tau<f^{m}, \phi_{j}>+\left(u_{h}^{m-1}, \phi_{j}\right) .
\end{array}
$$

Following the abstract Schwarz theory, presented, e.g., in [33], we consider a family of subspaces $V_{i} \subset V_{h}, i=0, \ldots, N$, and interpolation (or extension) operators $R_{i}^{T}: V_{i} \longrightarrow V_{h}$, and assume that $V_{h}$ admits the decomposition

$$
V_{h}=\sum_{i=0}^{N} R_{i}^{T} V_{i}
$$

We suppose that there exists a symmetric, continuous and coercive bilinear form $a: V \times V \longrightarrow \mathbf{R}$, such that

$$
b(u, v)=a(u, v)+\tilde{b}(u, v),
$$

with $\tilde{b}$ a nonlinear form, monotone and Lipschitz continuous with Lipschitz constant $\tilde{L}$. Nonlinear forms $b(u, v)$ with such a structure arise, e.g., in the field of computational electrocardiology, where research on parallel solvers for the associated nonlinear parabolic reactiondiffusion models (known as monodomain and bidomain models) is currently very active; see, e.g., $[25,13,30,22,24,21,23,28]$.

Since the bilinear form

$$
a_{\tau}(u, v)=(u, v)+\tau a(u, v)
$$

defines a scalar product on $V$, we can introduce local symmetric, positive definite bilinear forms $\tilde{a}_{\tau, i}: V_{i} \times V_{i} \longrightarrow \mathbf{R}$, and we make the standard three assumptions of the abstract Schwarz theory (see [33] for more details): 
- stable decomposition: there exists a constant $C_{0}$, such that every $u \in V_{h}$ admits a decomposition $u=\sum_{i=0}^{N} R_{i}^{T} u_{i}, u_{i} \in V_{i}, i=0, \ldots, N$ that satisfies

$$
\sum_{i=0}^{N} \tilde{a}_{\tau, i}\left(u_{i}, u_{i}\right) \leq C_{0}^{2} a_{\tau}(u, u)
$$

- strengthened Cauchy-Schwarz inequality: $\exists \epsilon_{i j} \in[0,1] i, j=1, \ldots, N$, such that $\forall u_{i} \in V_{i}, \forall u_{j} \in V_{j}$

$$
\left|a_{\tau}\left(R_{i}^{T} u_{i}, R_{j}^{T} u_{j}\right)\right| \leq \epsilon_{i j} a_{\tau}\left(R_{i}^{T} u_{i}, R_{i}^{T} u_{i}\right)^{1 / 2} a_{\tau}\left(R_{j}^{T} u_{j}, R_{j}^{T} u_{j}\right)^{1 / 2}
$$

(we denote by $\rho(\mathcal{E})$ the spectral radius of the matrix $\mathcal{E}=\left(\epsilon_{i j}\right)$ )

- local stability: there exists $\omega>0$, such that

$$
a_{\tau}\left(R_{i}^{T} u_{i}, R_{i}^{T} u_{i}\right) \leq \omega \tilde{a}_{\tau, i}\left(u_{i}, u_{i}\right), \forall u_{i} \in V_{i}, 0 \leq i \leq N .
$$

We define the "projection"-like operators $\widetilde{Q}_{i}: V_{h} \longrightarrow V_{i}$ by

$$
\tilde{a}_{\tau, i}\left(\widetilde{Q}_{i}(u), v_{i}\right)=\left(u, R_{i}^{T} v_{i}\right)+\tau b\left(u, R_{i}^{T} v_{i}\right), \forall v_{i} \in V_{i}, u \in V_{h},
$$

and their extensions $Q_{i}: V_{h} \longrightarrow R_{i}^{T} V_{i} \subset V_{h}$ defined by

$$
Q_{i}(u)=R_{i}^{T} \widetilde{Q}_{i}(u) \text { and } Q(u)=\sum_{i=0}^{N} Q_{i}(u)
$$

Let $\widetilde{A}_{\tau, i} \equiv\left(\tilde{a}_{\tau, i}\left(\phi_{j}, \phi_{l}\right)\right)_{j, l}$ be the matrix representation of the local bilinear form $\tilde{a}_{\tau, i}$.

LEMMA 3.1. The matrix form of $Q(u)$ is

$$
Q(u)=\mathcal{M}^{-1} B(u),
$$

where $\mathcal{M}=\left(\sum_{i=0}^{N} R_{i}^{T} \widetilde{A}_{\tau, i}^{-1} R_{i}\right)^{-1}$.

Proof. Let $\left\{\psi_{i}^{k}, k=1, \ldots, n_{i}\right\}$ be a basis of the local subspace $V_{i}$. Then $\widetilde{Q}_{i}(u) \in V_{i}$ can be written as $\widetilde{Q}_{i}(u)=\sum_{l} \widetilde{Q}_{i}(u)^{l} \psi_{i}^{l}$ and the two terms in (3.6) become

$$
\begin{aligned}
\tilde{a}_{\tau, i}\left(\widetilde{Q}_{i}(u), v_{i}\right) & =\tilde{a}_{\tau, i}\left(\sum_{l} \widetilde{Q}_{i}(u)^{l} \psi_{i}^{l}, \sum_{k} v_{i}^{k} \psi_{i}^{k}\right)=\sum_{l} \sum_{k} \widetilde{Q}_{i}(u)^{l} \tilde{a}_{\tau, i}\left(\psi_{i}^{l}, \psi_{i}^{k}\right) v_{i}^{k} \\
& =\sum_{k} v_{i}^{k} \sum_{l} \widetilde{A}_{\tau, i}^{k l} \widetilde{Q}_{i}(u)^{l}=v_{i}^{T} \widetilde{A}_{\tau, i} \widetilde{Q}_{i}(u),
\end{aligned}
$$

and

$$
\begin{aligned}
\left(u, R_{i}^{T} v_{i}\right)+\tau b\left(u, R_{i}^{T} v_{i}\right) & =\left(u, \sum_{l=1}^{n}\left(R_{i}^{T} v_{i}\right)^{l} \phi_{l}\right)+\tau b\left(u, \sum_{l=1}^{n}\left(R_{i}^{T} v_{i}\right)^{l} \phi_{l}\right) \\
& =\sum_{l=1}^{n}\left(R_{i}^{T} v_{i}\right)^{l}\left[\left(u, \phi_{l}\right)+\tau b\left(u, \phi_{l}\right)\right]=\sum_{l=1}^{n}\left(R_{i}^{T} v_{i}\right)^{l} B_{l} \\
& =\left(R_{i}^{T} v_{i}\right)^{T} B(u) .
\end{aligned}
$$


The definition of $\widetilde{Q}_{i}(u)$ implies

$$
v_{i}^{T} \widetilde{A}_{\tau, i} \widetilde{Q}_{i}(u)=\left(R_{i}^{T} v_{i}\right)^{T} B(u), \forall v_{i} \in V_{i}, u \in V_{h},
$$

and consequently

$$
\widetilde{A}_{\tau, i} \widetilde{Q}_{i}(u)=R_{i} B(u), \quad \forall u \in V_{h} .
$$

The matrix form of $\widetilde{Q}_{i}(u)$ is then

$$
\widetilde{Q}_{i}(u)=\widetilde{A}_{\tau, i}^{-1} R_{i} B(u) .
$$

Since $Q_{i}(u)=R_{i}^{T} \widetilde{Q}_{i}(u)$, the matrix form of $Q_{i}(u)$ is

$$
Q_{i}(u)=R_{i}^{T} \widetilde{A}_{\tau, i}^{-1} R_{i} B(u) .
$$

Hence,

$$
Q(u)=\sum_{i=0}^{N} Q_{i}(u)=\sum_{i=0}^{N} R_{i}^{T} \widetilde{A}_{\tau, i}^{-1} R_{i} B(u)=\mathcal{M}^{-1} B(u),
$$

where $\mathcal{M}=\left(\sum_{i=0}^{N} R_{i}^{T} \widetilde{A}_{\tau, i}^{-1} R_{i}\right)^{-1}$. .

REMARK 3.2. Since the matrix $\mathcal{M}$ is symmetric and positive definite, it defines the $\mathcal{M}$-norm $\|u\|_{\mathcal{M}}=\left(u^{T} \mathcal{M} u\right)^{1 / 2}$.

We define $\check{g}_{i}=Q_{i}\left(u^{*}\right)$, where $u^{*}$ is the exact solution of $(3.1)$, i.e: $B\left(u^{*}\right)=\hat{g}$. We consider the nonlinear system

$$
Q(u)=\check{g},
$$

where $\check{g}=\sum_{i=0}^{N} \check{g}_{i}$. As in the linear case (see [29, p. 150]), $\check{g}$ can be computed without knowing the exact solution $u^{*}$, since

$$
\check{g}=\sum_{i=0}^{N} \check{g}_{i}=\sum_{i=0}^{N} Q_{i}\left(u^{*}\right)=\sum_{i=0}^{N} R_{i}^{T} \widetilde{A}_{\tau, i} R_{i} B\left(u^{*}\right)=\mathcal{M}^{-1} B\left(u^{*}\right)=\mathcal{M}^{-1} \hat{g} .
$$

Using the matrix form of the nonlinear operator $Q$ and the definition of $\breve{g}$, it is straightforward to prove that the nonlinear system $B(u)=\hat{g}$ is equivalent to the nonlinear system $Q(u)=\check{g}$. In other words, we use the symmetric positive definite part as a preconditioner for the original nonlinear system. This idea has already been used by Cai and Xu [34] for nonsymmetric or indefinite problems. We can then define the Additive Schwarz-Richardson (ASR) algorithm for Problem (3.1).

ASR algorithm: given initial guesses $u^{0}, r^{0}=Q\left(u^{0}\right)-\check{g}$ and a stopping criterion, iterate for $k=0,1, \ldots$ until convergence

$$
\begin{array}{ll}
\text { solve the preconditioned system: } & \mathcal{M} r^{k}=B\left(u^{k}\right)-\hat{g}, \\
\text { update the solution: } & u^{k+1}=u^{k}-\lambda r^{k},
\end{array}
$$

where $\lambda$ is a properly chosen parameter; see Theorem 4.7.

We prove in the next section that the ASR iterations converge if $\lambda$ is chosen properly, and its convergence rate depends on the parameters $C_{0}, \epsilon_{i j}, \omega$ defined previously. 
4. An abstract convergence result. In order to prove our main result, Theorem 4.7, we need a few technical results established in the next few lemmas. We start by recalling a lemma due to Zhang [36], that plays an important role in proving the equivalence between $\|\cdot\|_{a_{\tau}}$ and $\|\cdot\|_{\mathcal{M}}$.

LEMma 4.1. Let $\widetilde{P}_{i}$ be the projection-like operator from $V$ onto $V_{i}$ defined by $\tilde{a}_{\tau, i}\left(\widetilde{P}_{i} u, v_{i}\right)=a_{\tau}\left(u, R_{i}^{T} v_{i}\right), v_{i} \in V_{i}$ and $P_{a d}=\sum_{i=0}^{N} R_{i}^{T} \widetilde{P}_{i}=\mathcal{M}^{-1} A_{\tau}$. Then

$$
a_{\tau}\left(P_{a d}^{-1} u, u\right)=\min _{\substack{u_{i} \in V_{i} \\ u=\sum R_{i}^{T} u_{i}}} \sum_{i=0}^{N} \tilde{a}_{\tau, i}\left(u_{i}, u_{i}\right) .
$$

LEMMA 4.2. The $\mathcal{M}-$ norm and $a_{\tau}-$ norm are equivalent, i.e.,

$$
\frac{1}{C_{0}^{2}}\|u\|_{\mathcal{M}}^{2} \leq\|u\|_{a_{\tau}}^{2} \leq 2 \omega(1+\rho(\mathcal{E}))\|u\|_{\mathcal{M}}^{2},
$$

where $C_{0}, \omega$ and $\rho(\mathcal{E})$ are the constants defined in the three assumptions of the abstract Schwarz theory, inequalities (3.3)-(3.5).

Proof. a) Lower bound. Using Lemma 4.1 and the stable decomposition assumption (3.3), we have

$$
\begin{aligned}
\|u\|_{\mathcal{M}}^{2} & =u^{T} \mathcal{M} u=u^{T} A_{\tau} A_{\tau}^{-1} \mathcal{M} u=a_{\tau}\left(A_{\tau}^{-1} \mathcal{M} u, u\right) \\
& =a_{\tau}\left(P_{a d}^{-1} u, u\right) \leq \sum_{i=0}^{N} \tilde{a}_{\tau, i}\left(u_{i}, u_{i}\right) \leq C_{0}^{2}\|u\|_{a_{\tau}}^{2} .
\end{aligned}
$$

b) Upper bound. Let $\bar{u}=\sum_{i=1}^{N} R_{i}^{T} u_{i}$. From the strengthened Cauchy-Schwarz inequality (3.4) and the local stability assumption (3.5), it follows that

$$
\begin{aligned}
a_{\tau}(\bar{u}, \bar{u}) & =\sum_{i, j=1}^{N} a_{\tau}\left(R_{i}^{T} u_{i}, R_{j}^{T} u_{j}\right) \leq \sum_{i, j=1}^{N} \epsilon_{i j} a_{\tau}\left(R_{i}^{T} u_{i}, R_{i}^{T} u_{i}\right)^{1 / 2} a_{\tau}\left(R_{j}^{T} u_{j}, R_{j}^{T} u_{j}\right)^{1 / 2} \\
& \leq \rho(\mathcal{E}) \sum_{i=1}^{N} a_{\tau}\left(R_{i}^{T} u_{i}, R_{i}^{T} u_{i}\right) \leq \rho(\mathcal{E}) \omega \sum_{i=1}^{N} \tilde{a}_{\tau, i}\left(u_{i}, u_{i}\right) .
\end{aligned}
$$

Each element $u \in V_{h}$ can be written as $u=R_{0}^{T} u_{0}+\bar{u}$. Using the bilinearity of $a_{\tau}$, the local stability assumption and the last bound, we have

$$
\begin{aligned}
a_{\tau}(u, u) & =a_{\tau}\left(R_{0}^{T} u_{0}+\bar{u}, R_{0}^{T} u_{0}+\bar{u}\right)=a_{\tau}\left(R_{0}^{T} u_{0}, R_{0}^{T} u_{0}\right)+a_{\tau}(\bar{u}, \bar{u})+2 a_{\tau}\left(R_{0}^{T} u_{0}, \bar{u}\right) \\
& \leq \omega \tilde{a}_{\tau, 0}\left(u_{0}, u_{0}\right)+\rho(\mathcal{E}) \omega \sum_{i=1}^{N} \tilde{a}_{\tau i}\left(u_{i}, u_{i}\right)+2 a_{\tau}\left(R_{0}^{T} u_{0}, \bar{u}\right) .
\end{aligned}
$$

Since $2 a_{\tau}\left(R_{0}^{T} u_{0}, \bar{u}\right) \leq 2 a_{\tau}\left(R_{0}^{T} u_{0}, R_{0}^{T} u_{0}\right)^{1 / 2} a_{\tau}(\bar{u}, \bar{u})^{1 / 2} \leq a_{\tau}\left(R_{0}^{T} u_{0}, R_{0}^{T} u_{0}\right)+a_{\tau}(\bar{u}, \bar{u})$, we obtain

$$
a_{\tau}(u, u) \leq 2 \omega(1+\rho(\mathcal{E})) \sum_{i=0}^{N} \tilde{a}_{\tau, i}\left(u_{i}, u_{i}\right), \forall u_{i} \in V_{i}, u=\sum_{i=0}^{N} R_{i}^{T} u_{i} .
$$


Taking the minimum over the all decompositions of $u$ and using Lemma 4.1, we conclude

$$
\begin{gathered}
a_{\tau}(u, u) \leq 2 \omega(1+\rho(\mathcal{E})) \min _{\substack{u_{i} \in V_{i} \\
u}} \sum_{i} \tilde{a}_{\tau, i}\left(u_{i}, u_{i}\right)=2 \omega(1+\rho(\mathcal{E})) a_{\tau}\left(P_{a d}^{-1} u, u\right) \\
=2 \omega(1+\rho(\mathcal{E}))\|u\|_{\mathcal{M}_{i}}^{2} \text {. } \square \\
\text { LEMMA 4.3. There exists a constant } \delta_{0}=\frac{1}{C_{0}^{2}}>0 \text {, such that }
\end{gathered}
$$

$$
(Q(u+z)-Q(u), z)_{\mathcal{M}} \geq \delta_{0}\|z\|_{\mathcal{M}}^{2}, \forall u, z \in V_{h} .
$$

Proof. The definition of $Q(u)$, the linearity of $b$ in the second argument, the strict monotonicity of $\tilde{b}$, and the lower bound of Lemma 4.2 imply

$$
\begin{aligned}
(Q(u+z)-Q(u), z)_{\mathcal{M}} & =z^{T} \mathcal{M}(Q(u+z)-Q(u)) \\
& =z^{T} \mathcal{M} \mathcal{M}^{-1}(B(u+z)-B(u)) \\
& =z^{T}(B(u+z)-B(u)) \\
& =\sum_{j=1}^{n} z_{j}\left(b_{j}(u+z)-b_{j}(u)\right) \\
& =\sum_{j=1}^{n} z_{j}\left[\left(u+z, \phi_{j}\right)+\tau b\left(u+z, \phi_{j}\right)-\left(u, \phi_{j}\right)-\tau b\left(u, \phi_{j}\right)\right] \\
& =\left(z, \sum_{j=1}^{n} z_{j} \phi_{j}\right)+\tau\left[b\left(u+z, \sum_{j=1}^{n} z_{j} \phi_{j}\right)-b\left(u, \sum_{j=1}^{n} z_{j} \phi_{j}\right)\right] \\
& =(z, z)+\tau[b(u+z, z)-b(u, z)] \\
& =\|z\|_{a_{\tau}}^{2}+\tau[\tilde{b}(u+z, z)-\tilde{b}(u, z)] \geq\|z\|_{a_{\tau}}^{2} \\
& \geq \frac{1}{C_{0}^{2}}\|z\|_{\mathcal{M}}^{2} .
\end{aligned}
$$

Using a particular decomposition of $z$, we are able to give another proof of this lemma. Since $P_{\text {ad }}$ is invertible (see [33, Lemma 2.5]), we can decompose $z \in V_{h}$ as $z=\sum_{j} R_{j}^{T} z_{j}$, where

$$
\begin{aligned}
z_{j} & =\widetilde{P}_{j} P_{\mathrm{ad}}^{-1} z \\
a_{\tau}(z, z) & =a_{\tau}(u+z, z)-a_{\tau}(u, z)=\sum_{j}\left[a_{\tau}\left(u+z, R_{j}^{T} z_{j}\right)-a_{\tau}\left(u, R_{j}^{T} z_{j}\right)\right] \\
& =\sum_{j}\left[\tilde{a}_{\tau j}\left(\widetilde{Q}_{j}(u+z), z_{j}\right)-\tilde{a}_{\tau j}\left(\widetilde{Q}_{j}(u), z_{j}\right)\right]-\sum_{j} \tau\left[\tilde{b}\left(u+z, R_{j}^{T} z_{j}\right)-\tilde{b}\left(u, R_{j}^{T} z_{j}\right)\right] \\
& =\sum_{j}\left[\tilde{a}_{\tau j}\left(\widetilde{Q}_{j}(u+z), z_{j}\right)-\tilde{a}_{\tau j}\left(\widetilde{Q}_{j}(u), z_{j}\right)\right]-\tau[\tilde{b}(u+z, z)-\tilde{b}(u, z)] .
\end{aligned}
$$

Using the monotonicity of $\tilde{b}$, we can write

$$
a_{\tau}(z, z) \leq \sum_{j} \tilde{a}_{\tau j}\left(\widetilde{Q}_{j}(u+z)-\tilde{Q}_{j}(u), z_{j}\right) .
$$


By the definition of $z_{j}, \widetilde{P}_{j}=\widetilde{A}_{\tau j}^{-1} R_{j} A_{\tau}, P_{\text {ad }}=\mathcal{M}^{-1} A_{\tau}$, and we have

$$
\begin{aligned}
\|z\|_{a_{\tau}}^{2} & \leq \sum_{j} \tilde{a}_{\tau j}\left(\widetilde{Q}_{j}(u+z)-\widetilde{Q}_{j}(u), z_{j}\right)=\sum_{j} z_{j}^{T} \widetilde{A}_{\tau j}\left[\widetilde{Q}_{j}(u+z)-\widetilde{Q}_{j}(u)\right] \\
& =\sum_{j} z^{T}\left(P_{\mathrm{ad}}^{-1}\right)^{T} \widetilde{P}_{j}^{T} \widetilde{A}_{\tau j} \widetilde{A}_{\tau j}^{-1} R_{j}[B(u+z)-B(u)] \\
& =z^{T}\left(P_{\mathrm{ad}}^{-1}\right)^{T}\left(\sum_{j} \widetilde{P}_{j}^{T} R_{j}\right)[B(u+z)-B(u)] \\
& =z^{T}\left(P_{\mathrm{ad}}^{-1}\right)^{T}\left(\sum_{j} A_{\tau}^{T} R_{j}^{T}\left(\widetilde{A}_{\tau j}^{-1}\right)^{T} R_{j}\right)[B(u+z)-B(u)] \\
& =z^{T}\left(P_{\mathrm{ad}}^{-1}\right)^{T} A_{\tau} \mathcal{M}^{-1}[B(u+z)-B(u)]=z^{T}\left(A_{\tau}^{-1} \mathcal{M}\right)^{T} A_{\tau} \mathcal{M}^{-1}[B(u+z)-B(u)] \\
& =z^{T} \mathcal{M}^{T}\left(A_{\tau}^{-1}\right)^{T} A_{\tau} \mathcal{M}^{-1}[B(u+z)-B(u)]=z^{T} \mathcal{M}[Q(u+z)-Q(u)] \\
& =(z, Q(u+z)-Q(u))_{\mathcal{M}} .
\end{aligned}
$$

We conclude the proof by using Lemma 4.2. $\square$

LEMMA 4.4. For $i=0,1, \ldots, N$, we have that

a) $\left\|Q_{i}(u+z)-Q_{i}(u)\right\|_{a_{\tau}}^{2} \leq \omega\left[a_{\tau}\left(z, Q_{i}(u+z)-Q_{i}(u)\right)+\tau \tilde{b}\left(u+z, Q_{i}(u+z)-Q_{i}(u)\right)\right.$

$$
\left.-\tau \tilde{b}\left(u, Q_{i}(u+z)-Q_{i}(u)\right)\right]
$$

b) $\left\|Q_{i}(u+z)-Q_{i}(u)\right\|_{a_{\tau}} \leq \omega(1+c \tilde{L})\|z\|_{a_{\tau}}$.

Proof. a) Using the definition of $Q_{i}$, the local stability assumption, the definition of $\widetilde{Q}_{i}$, the linearity of $a_{\tau}$, and the Lipschitz continuity of $\tilde{b}$, we have,

$$
\begin{aligned}
\| Q_{i}(u+z)- & Q_{i}(u) \|_{a_{\tau}}^{2} \\
& =a_{\tau}\left(Q_{i}(u+z)-Q_{i}(u), Q_{i}(u+z)-Q_{i}(u)\right) \\
& =a_{\tau}\left(R_{i}^{T}\left(\widetilde{Q}_{i}(u+z)-\widetilde{Q}_{i}(u)\right), R_{i}^{T}\left(\widetilde{Q}_{i}(u+z)-\widetilde{Q}_{i}(u)\right)\right) \\
\leq & \omega \tilde{a}_{\tau}\left(\widetilde{Q}_{i}(u+z)-\widetilde{Q}_{i}(u), \widetilde{Q}_{i}(u+z)-\widetilde{Q}_{i}(u)\right) \\
= & \omega\left[\left(u+z, R_{i}^{T}\left(\widetilde{Q}_{i}(u+z)-\widetilde{Q}_{i}(u)\right)\right)+\tau b\left(u+z, R_{i}^{T}\left(\widetilde{Q}_{i}(u+z)-\widetilde{Q}_{i}(u)\right)\right)\right. \\
& \left.\quad-\left(u, R_{i}^{T}\left(\widetilde{Q}_{i}(u+z)-\widetilde{Q}_{i}(u)\right)\right)-\tau b\left(u, R_{i}^{T}\left(\widetilde{Q}_{i}(u+z)-\widetilde{Q}_{i}(u)\right)\right)\right] \\
= & \omega\left[\left(z, Q_{i}(u+z)-Q_{i}(u)\right)+\tau a\left(z, Q_{i}(u+z)-Q_{i}(u)\right)+\right. \\
& \left.\tau \tilde{b}\left(u+z, Q_{i}(u+z)-Q_{i}(u)\right)-\tau \tilde{b}\left(u, Q_{i}(u+z)-Q_{i}(u)\right)\right] .
\end{aligned}
$$

b) By the Cauchy-Schwarz inequality, we have

$$
\begin{aligned}
\| Q_{i}(u+z) & -Q_{i}(u) \|_{a_{\tau}}^{2} \\
& \leq \omega\left[\|z\|_{a_{\tau}}\left\|Q_{i}(u+z)-Q_{i}(u)\right\|_{a_{\tau}}+\tau \tilde{L}\|z\| \cdot\left\|Q_{i}(u+z)-Q_{i}(u)\right\|\right] .
\end{aligned}
$$


The coercivity of the bilinear form $a(\cdot, \cdot)$ implies that $\tau\|z\|^{2} \leq c \tau a(z, z) \leq c a_{\tau}(z, z)$ and consequently we have $\left\|Q_{i}(u+z)-Q_{i}(u)\right\|_{a_{\tau}}^{2} \leq \omega(1+c \tilde{L})\|z\|_{a_{\tau}}\left\|Q_{i}(u+z)-Q_{i}(u)\right\|_{a_{\tau}}$.

LEMMA 4.5. There exists a constant $\breve{\delta}_{1}^{2}=2 \omega^{2}\left(\rho(\mathcal{E})^{2}+1\right)(1+c \tilde{L})^{2}>0$, such that

$$
\|Q(u+z)-Q(u)\|_{a_{\tau}} \leq \breve{\delta}_{1}\|z\|_{a_{\tau}}, \forall u, v \in V_{h} .
$$

Proof. Define $\bar{Q}(u)=\sum_{i=1}^{N} Q_{i}(u)$, as in the proof of the upper bound of Lemma 4.2. The bilinearity of $a_{\tau}$, the definition of $Q_{i}(u)$, and the strengthened Cauchy-Schwarz inequality, imply that

$$
\|\bar{Q}(u+z)-\bar{Q}(u)\|_{a_{\tau}}^{2} \leq \rho(\mathcal{E}) \sum_{i=1}^{N} a_{\tau}\left(Q_{i}(u+z)-Q_{i}(u), Q_{i}(u+z)-Q_{i}(u)\right) .
$$

From Lemma 4.4, we obtain

$$
\begin{aligned}
& \|\bar{Q}(u+z)-\bar{Q}(u)\|_{a_{\tau}}^{2} \\
& \leq \omega \rho(\mathcal{E}) \sum_{i=1}^{N}\left[a_{\tau}\left(z, Q_{i}(u+z)-Q_{i}(u)\right)+\right. \\
& \left.+\tau \tilde{b}\left(u+z, Q_{i}(u+z)-Q_{i}(u)\right)-\tau \tilde{b}\left(u, Q_{i}(u+z)-Q_{i}(u)\right)\right] \\
& \leq \rho(\mathcal{E}) \omega\left[a_{\tau}(z, \bar{Q}(u+z)-\bar{Q}(u))+\tau \tilde{b}(u+z, \bar{Q}(u+z)-\bar{Q}(u))-\tau \tilde{b}(u, \bar{Q}(u+z)-\bar{Q}(u))\right] \\
& \leq \rho(\mathcal{E}) \omega(1+c \tilde{L})\|z\|_{a_{\tau}}\|\bar{Q}(u+z)-\bar{Q}(u)\|_{a_{\tau}} .
\end{aligned}
$$

Hence,

$$
\|\bar{Q}(u+z)-\bar{Q}(u)\|_{a_{\tau}} \leq \rho(\mathcal{E}) \omega(1+c \tilde{L})\|z\|_{a_{\tau}} .
$$

Since $Q(u)=Q_{0}(u)+\bar{Q}(u)$, relation (4.2) and Lemma 4.4 give us

$$
\begin{aligned}
& \|Q(u+z)-Q(u)\|_{a_{\tau}}^{2} \leq 2\left\|Q_{0}(u+z)-Q_{0}(u)\right\|_{a_{\tau}}^{2}+2\|\bar{Q}(u+z)-\bar{Q}(u)\|_{a_{\tau}}^{2} \\
& \leq 2[\omega(1+\tau \tilde{L})]^{2}\|z\|_{a_{\tau}}^{2}+2[\rho(\mathcal{E}) \omega(1+\tau \tilde{L})]^{2}\|z\|_{a_{\tau}}^{2}=2 \omega^{2}(1+\tau \tilde{L})^{2}\left(1+\rho(\mathcal{E})^{2}\right)\|z\|_{a_{\tau}}^{2} .
\end{aligned}
$$

LEMMA 4.6. There exists a constant $\delta_{1}=C_{0}^{2} \breve{\delta}_{1}^{2} 2 \omega(1+\rho(\mathcal{E}))$, such that

$$
\|Q(u+z)-Q(u)\|_{\mathcal{M}}^{2} \leq \delta_{1}\|z\|_{\mathcal{M}}^{2} .
$$

Proof. This bound is a direct consequence of Lemma 4.2 and Lemma 4.5:

$$
\begin{aligned}
\|Q(u+z)-Q(u)\|_{\mathcal{M}}^{2} & \leq C_{0}^{2}\|Q(u+z)-Q(u)\|_{a_{\tau}}^{2} \\
& \leq C_{0}^{2} \breve{\delta}_{1}^{2}\|z\|_{a_{\tau}}^{2} \\
& \leq C_{0}^{2} \breve{\delta}_{1}^{2} 2 \omega(1+\rho(\mathcal{E}))\|z\|_{\mathcal{M}}^{2} .
\end{aligned}
$$


Using these lemmas, we are now in a position to prove the main convergence result for our ASR method.

THEOREM 4.7. If $0<\lambda<2 \delta_{0} / \delta_{1}^{2}$, then the ASR method for Problem (3.8) converges, i.e.,

$$
\left\|u^{k}-u^{*}\right\|_{\mathcal{M}}^{2} \leq P(\lambda)^{k}\left\|u^{0}-u^{*}\right\|_{\mathcal{M}}^{2},
$$

where $P(\lambda)=1-2 \lambda \delta_{0}+\lambda^{2} \delta_{1}^{2}$ and $\delta_{0}, \delta_{1}$ are the constants defined in Lemma 4.3 and Lemma 4.6, respectively.

Proof. Let $e^{k}=u^{k}-u^{*}$ and $r^{k}=Q\left(u^{k}\right)-Q\left(u^{*}\right)$ be the error and the residual at step $k$. Then

$$
e^{k+1}=u^{k+1}-u^{*}=u^{k}-\lambda r^{k}-u^{*}=e^{k}-\lambda r^{k},
$$

and

$$
\begin{aligned}
\left\|e^{k+1}\right\|_{\mathcal{M}}^{2} & =\left(e^{k+1}, e^{k+1}\right)_{\mathcal{M}}\left(e^{k}-\lambda r^{k}, e^{k}-\lambda r^{k}\right)_{\mathcal{M}} \\
& =\left\|e^{k}\right\|_{\mathcal{M}}^{2}-2 \lambda\left(e^{k}, r^{k}\right)_{\mathcal{M}}+\lambda^{2}\left\|r^{k}\right\|_{\mathcal{M}}^{2} .
\end{aligned}
$$

Lemma 4.3 with $u=u^{*}$ and $z=u^{k}-u^{*}$ yields

$$
\begin{aligned}
-\left(e^{k}, r^{k}\right)_{\mathcal{M}} & =-\left(u^{k}-u^{*}, Q\left(u^{k}\right)-Q\left(u^{*}\right)\right)_{\mathcal{M}} \\
& =-\left(u^{k}-u^{*}, Q\left(u^{k}-u^{*}+u^{*}\right)-Q\left(u^{*}\right)\right)_{\mathcal{M}} \\
& \leq-\delta_{0}\left\|e^{k}\right\|_{\mathcal{M}}^{2} .
\end{aligned}
$$

Using Lemma 4.6 with $u=u^{*}$ and $z=u^{k}-u^{*}$, we obtain

$$
\left\|r_{k}\right\|_{\mathcal{M}}^{2}=\left\|Q\left(u^{k}\right)-Q\left(u^{*}\right)\right\|_{\mathcal{M}}^{2}=\left\|Q\left(u^{k}-u^{*}+u^{*}\right)-Q\left(u^{*}\right)\right\|_{\mathcal{M}}^{2} \leq \delta_{1}^{2}\left\|e^{k}\right\|_{\mathcal{M}}^{2} .
$$

Therefore

$$
\left\|e^{k+1}\right\|_{\mathcal{M}}^{2} \leq\left(1-2 \lambda \delta_{0}+\lambda^{2} \delta_{1}^{2}\right)\left\|e^{k}\right\|_{\mathcal{M}}^{2}=P(\lambda)\left\|e^{k}\right\|_{\mathcal{M}}^{2} .
$$

If we choose $0<\lambda<\frac{2 \delta_{0}}{\delta_{1}^{2}}$, then $P(\lambda)<1$, and we obtain the convergence of the ASR method.

REMARK 4.8. $P(\lambda)$ attains its minimum at $\lambda_{\min }=\frac{\delta_{0}}{\delta_{1}^{2}}$ and $P\left(\lambda_{\min }\right)=1-\frac{\delta_{0}^{2}}{\delta_{1}^{2}}<1$.

REMARK 4.9. If we drop the coarse space $V_{0}$ and define $Q(u)=\sum_{i=1}^{N} Q_{i}(u)$, the ASR algorithm is convergent. It is easy to see that in this case $\delta_{0}=\frac{1}{C_{0}^{2}}$ and $\delta_{1}=C_{0} \rho(\mathcal{E}) \omega(1+c \tilde{L})$.

REMARK 4.10. The ASR performance depends on the choice of the parameter $\lambda$ in the Richardson iteration. This parameter can be chosen:

i) theoretically, by approximately minimizing the quadratic function $P(\lambda)$ in Theorem 4.7 if the constants $\delta_{0}$ and $\delta_{1}$ can be estimated;

ii) numerically, by running a few tests cases (as we have done in Section 6, Figure 6.1) and selecting a value which gives an approximate minimum of the ASR iteration count;

iii) automatically, by using one of the step-length strategies available in the literature of numerical optimization, such as the one described in [26]; see the results reported in Section 6, Table 6.2.

We remark that the numerical results of Figure 6.1 show that the ASR iteration count is not very sensitive to the choice of $\lambda$ near the minimum, but only near the endpoints of the convergence interval. 
5. Convergence estimates for parabolic problem. Our additive Schwarz preconditioner is build as in the linear case. We partition the domain $\Omega$ into shape regular nonoverlapping subdomains $\Omega_{i}, 1 \leq i \leq N$, of characteristic diameter $H$, defining a shape-regular coarse mesh $\mathcal{T}_{H}$. Each subdomain $\Omega_{i}$ is extended to a larger one, $\Omega_{i}^{\prime}$, by adding the elements of the fine mesh $\mathcal{T}_{h}$ within a distance $\delta_{i}$ from its boundary. We assume that the partition $\left\{\Omega_{i}^{\prime}\right\}$ satisfies the finite covering assumption (see, e.g., [33]), and we denote by $\delta=\max _{i} \delta_{i}$ the overlap size. Using the above decomposition, a one-level method is defined by the local spaces $V_{i}=\left\{v \in H_{0}^{1}\left(\Omega_{i}^{\prime}\right)|v|_{T}\right.$ is linear, $\left.\forall T \in T_{h, i}\right\}, 1 \leq i \leq N$, and the local bilinear forms $\tilde{a}_{\tau, i}\left(u_{i}, v_{i}\right)=a_{\tau}\left(R_{i}^{T} u_{i}, R_{i}^{T} v_{i}\right), \forall u_{i}, v_{i} \in V_{i}$, with interpolation operators $R_{i}^{T}: V_{i} \longrightarrow V, 1 \leq i \leq N$. We then build a two-level algorithm by defining the coarse finite element space $V_{0}=\left\{v \in H_{0}^{1}(\Omega) \mid v\right.$ is continuous and $\left.v\right|_{T}$ is linear, $\left.\forall T \in T_{H}\right\}$ and the operator $R_{0}^{T}$, which interpolates the coarse functions onto the fine mesh.

We consider the variational parabolic problem: given $u\left(t_{0}, x\right)=u_{0}(x)$ and right-hand side $G$, find $u(t) \in H^{1}(\Omega)$, such that $\forall t \in\left(t_{0}, T\right)$,

$$
\left(\frac{\partial u}{\partial t}, v\right)+a(u, v)+(F(u), v)=(G, v)
$$

where

$$
a(u, v)=\int_{\Omega} \sum_{i, j=1}^{d} a_{i j} \frac{\partial u}{\partial x_{i}} \frac{\partial v}{\partial x_{j}} d x,
$$

with $a_{i j} \in C^{1}(\Omega)$, such that $a_{i j}(x)=a_{j i}(x), \forall x \in \Omega \subset \mathbf{R}^{d}$ for all $i, j=1, \ldots, d$ and $F$ a monotone nonlinear function.

The abstract convergence result of Theorem 4.7 now can be applied to get explicit bounds in terms of the discretization parameters. For simplicity, we consider the simplest case, where we use exact solvers (i.e., $\left.\tilde{a}_{\tau, i}\left(u_{i}, v_{i}\right)=a_{\tau}\left(R_{i}^{T} u_{i}, R_{i}^{T} v_{i}\right), \forall u_{i}, v_{i} \in V_{i}\right)$, so that $\omega=1$ in the local stability assumption. We also assume that there are at most $N^{c}$ nonzero elements in each row of $\mathcal{E} \leq N^{c}$, so that $\rho(\mathcal{E}) \leq N^{c}$ in the strengthened Cauchy-Schwarz inequality; see [33, Lemma 2.10]. Therefore, we need only to bound the constant $C_{0}$ in the stable decomposition assumption.

LEMMA 5.1. The stable decomposition constant $C_{0}$ can be bounded by

$$
\begin{aligned}
& \text { a) } C_{0}^{2} \leq C \max \left\{1+\frac{H}{\delta}, 1+\frac{\tau}{H \delta}\right\} \text {, in the one-level case, } \\
& \text { b) } C_{0}^{2} \leq C\left(1+\frac{H}{\delta}\right) \text {, in the two-level case, }
\end{aligned}
$$

where $C$ is a constant independent of $H, h$ and $\delta$.

Proof. a) One-level case. Following the classical proof for the linear elliptic case (see, e.g., [33]), we define $u_{i}=R_{i}\left(I^{h}\left(\theta_{i} u\right)\right), i=1, \ldots, N$, where $\left\{\theta_{i} \in W^{1, \infty}, 1 \leq i \leq N\right\}$ defines a partition of unity and $I^{h}$ is the nodal piecewise linear interpolant on the fine mesh $\mathcal{T}_{h}$. By the approximations properties of $I^{h}$ and the small overlap lemma (see [33], Lemma 3.10 and eqs.(3.20) - (3.23) or the original Lemma 3.1 in [16]), we obtain

$$
\left|R_{i}^{T} u_{i}\right|_{H^{1}(\Omega)}^{2} \equiv\left|I^{h}\left(\theta_{i} u\right)\right|_{H^{1}\left(\Omega_{i}^{\prime}\right)}^{2} \leq C\left(1+\frac{H}{\delta}\right)|u|_{H^{1}\left(\Omega_{i}^{\prime}\right)}^{2}+\frac{1}{H_{i} \delta_{i}}\|u\|_{L^{2}\left(\Omega_{i}^{\prime}\right)}^{2} .
$$

The equivalence between the $L^{2}(\Omega)$-norm and the discrete $L^{2}(\Omega)$-norm implies that

$$
\left(R_{i}^{T} u_{i}, R_{i}^{T} u_{i}\right)_{L^{2}(\Omega)} \equiv\left\|I^{h}\left(\theta_{i} u\right)\right\|_{L^{2}\left(\Omega_{i}^{\prime}\right)}^{2} \leq C\|u\|_{L^{2}\left(\Omega_{i}^{\prime}\right)}^{2} .
$$


Using the coercivity of $a(\cdot, \cdot),(5.2),(5.3)$, and the finite covering assumption, we have

$$
\begin{aligned}
\sum_{i=1}^{N} \tilde{a}_{\tau, i}\left(u_{i}, u_{i}\right) & =\sum_{i=1}^{N} a_{\tau}\left(R_{i}^{T} u_{i}, R_{i}^{T} u_{i}\right)=\sum_{i=1}^{N}\left[\left(R_{i}^{T} u_{i}, R_{i}^{T} u_{i}\right)_{L^{2}(\Omega)}+\tau a\left(R_{i}^{T} u_{i}, R_{i}^{T} u_{i}\right)\right] \\
& \leq C \sum_{i=1}^{N}\left[\left(R_{i}^{T} u_{i}, R_{i}^{T} u_{i}\right)_{L^{2}(\Omega)}+\tau\left|R_{i}^{T} u_{i}\right|_{H^{1}(\Omega)}^{2}\right] \\
& \leq C \sum_{i=1}^{N}\left\{\|u\|_{L^{2}\left(\Omega_{i}^{\prime}\right)}^{2}+\tau\left[\left(1+\frac{H}{\delta}\right)|u|_{H^{1}\left(\Omega_{i}^{\prime}\right)}^{2}+\frac{1}{H \delta}\|u\|_{L^{2}\left(\Omega_{i}^{\prime}\right)}^{2}\right]\right\} \\
& \leq C \sum_{i=1}^{N}\left[\left(1+\frac{\tau}{H \delta}\right)\|u\|_{L^{2}\left(\Omega_{i}^{\prime}\right)}^{2}+\tau\left(1+\frac{H}{\delta}\right)|u|_{H^{1}\left(\Omega_{i}^{\prime}\right)}^{2}\right] \\
& \leq C\left(1+\frac{\tau}{H \delta}\right)\|u\|_{L^{2}(\Omega)}^{2}+\tau\left(1+\frac{H}{\delta}\right)|u|_{H^{1}(\Omega)}^{2} .
\end{aligned}
$$

The coercivity of the bilinear form $a(\cdot, \cdot)$ implies that $|u|_{H^{1}(\Omega)}^{2} \leq C a(u, u)$ and therefore

$$
\begin{aligned}
\sum_{i=1}^{N} \tilde{a}_{\tau, i}\left(u_{i}, u_{i}\right) & \leq C\left[\left(1+\frac{\tau}{H \delta}\right)\|u\|_{L^{2}(\Omega)}^{2}+\tau\left(1+\frac{H}{\delta}\right) a(u, u)\right] \\
& \leq C \max \left\{1+\frac{H}{\delta}, 1+\frac{\tau}{H \delta}\right\}\left[\|u\|_{L^{2}(\Omega)}^{2}+\tau a(u, u)\right] .
\end{aligned}
$$

b) Two-level case. Suppose that the coarse mesh is quasi-uniform. Then the bound for $C_{0}^{2}$ can be obtained as in [33] by letting $I^{H}: L^{2}(\Omega) \longrightarrow V_{0}$ be the $L^{2}$-projection of $u,\left(I^{H} u, v\right)_{L^{2}(\Omega)}=(u, v), \forall u \in L^{2}(\Omega), v \in V_{0}$, and $u_{0}=I^{H} u, w=u-I^{h} u_{0}$, $u_{i}=I^{h}\left(\theta_{i} w\right)$. $\mathrm{Q}$

REMARK 5.2. The bound a) of Lemma 5.1 implies that if the ratio $\tau /(H \delta)$ is small enough, then a constant upper bound $C_{0}^{2} \leq C\left(1+\frac{H}{h}\right)$ still holds for the one-level ASR algorithm.

REMARK 5.3. In case of small overlap $\delta=h$, the estimates of Lemma 5.1 become

a) $C_{0}^{2} \leq C \max \left\{1+\frac{H}{h}, 1+\frac{\tau}{H h}\right\}$, in the one-level case,

b) $C_{0}^{2} \leq C\left(1+\frac{H}{h}\right)$, in the two-level case.

Therefore, in scaled speed-up tests with constant ratio $H / h$ only the two-level ASR algorithm is scalable, since the term $\tau /(H h)$ asymptotically dominates the one-level bound for any fixed value of $\tau$. Nevertheless, for a moderate number of subdomains (i.e., for $1 / H$ small enough), the one-level bound is dominated by the first term $1+\frac{H}{h}$, which yields a "temporary" scalability; see Figure 6.2.

REMARK 5.4. In case of generous overlap $\delta=C H$, the estimates of Lemma 5.1 agree with the estimates obtained by Cai $[7,8]$ for linear parabolic problems. In such case, Cai proved that if $\tau / H^{2}$ is small enough, then the one-level Schwarz algorithm is scalable and the two-level algorithm satisfies an optimal constant bound. Lemma 5.1 extends these estimates to nonlinear parabolic problems and to the case of variable overlap. 
TABLE 6.1

Comparison of ASR and Linearly Implicit Euler methods (GMRES with AS preconditioner): scaled speed-up test with fixed subdomain size $H / h=4$, small overlap size $\delta=h$, and increasing number of subdomains $N$ (and nodes). iter $=$ iteration counts, $c p u=$ cpu times, err $=$ relative errors with exact solution.

\begin{tabular}{|c|c|c|c|c|c|c|c|c|c|c|c|}
\hline \multirow[b]{3}{*}{$\mathrm{N}$} & \multicolumn{4}{|c|}{ ASR with $\lambda=0.4$} & \multicolumn{6}{|c|}{ Lin. Impl. Euler: GMRES with AS prec. } & \multirow[b]{3}{*}{ err } \\
\hline & \multicolumn{2}{|c|}{ one-level } & \multicolumn{2}{|c|}{ two-levels } & \multicolumn{2}{|c|}{ one-level } & \multicolumn{2}{|c|}{ one-level, rest $=20$} & \multicolumn{2}{|c|}{ two-levels } & \\
\hline & iter & $\mathrm{cpu}$ & iter & $\mathrm{cpu}$ & iter & cpu & iter & $\mathrm{cpu}$ & iter & cpu & \\
\hline $2 \times 2$ & 40 & 0.07 & 43 & 0.07 & 10 & 0.09 & 10 & 0.10 & 11 & 0.07 & $6.76 \mathrm{e}-3$ \\
\hline $4 \times 4$ & 70 & 0.57 & 36 & 0.29 & 17 & 0.15 & 17 & 0.20 & 16 & 0.15 & $1.67 \mathrm{e}-3$ \\
\hline $6 \times 6$ & 123 & 2.88 & 35 & 0.87 & 20 & 0.59 & 20 & 0.84 & 17 & 0.49 & $7.44 \mathrm{e}-4$ \\
\hline $8 \times 8$ & 197 & 11.20 & 36 & 2.15 & 23 & 1.76 & $20+5$ & 2.93 & 16 & 1.26 & $4.18 \mathrm{e}-4$ \\
\hline $10 \times 10$ & 293 & 36.80 & 36 & 4.61 & 26 & 4.48 & $20+7$ & 7.78 & 16 & 3.02 & $2.67 \mathrm{e}-4$ \\
\hline $12 \times 12$ & 410 & 99.12 & 36 & 9.07 & 29 & 11.31 & $20+9$ & 18.54 & 16 & 6.63 & $1.86 \mathrm{e}-4$ \\
\hline $14 \times 14$ & 549 & 236.93 & 36 & 16.25 & 31 & 29.12 & $20+14$ & 51.33 & 16 & 15.69 & $1.36 \mathrm{e}-4$ \\
\hline $16 \times 16$ & 709 & 511.79 & 36 & 27.08 & 34 & 77.29 & $20+16$ & 128.71 & 16 & 37.42 & $1.04 \mathrm{e}-4$ \\
\hline $18 \times 18$ & 891 & 1013.27 & 36 & 43.18 & 36 & 162.50 & $20+18$ & 188.71 & 16 & 76.17 & $8.26 e-5$ \\
\hline
\end{tabular}

6. Numerical results. In this section, we report the results of MATLAB numerical experiments with the ASR method applied to the nonlinear parabolic problem (5.1) with the linear elliptic bilinear form and the nonlinear function

$$
a(u, v)=\sum_{i}^{N} \int_{\Omega_{i}} \sigma_{i} \nabla u \nabla v d x, \quad f(u)=0.5 u+u^{3} .
$$

The elliptic coefficients $\sigma_{i}$ are equal to 1 in Tables 6.2-6.4, while they are piecewise constant with jump discontinuities across subdomain boundaries in the last tests of Figures 6.3 and 6.4. The domain is the unit square $\Omega$ and the right-hand side $g$ is chosen so that $u^{*}(t, x)=$ $t \sin (\pi x) \sin (\pi y)$ is the exact solution when $\sigma_{i}=1$. We consider $t_{0}=0, u_{0}(x)=0$, and we compute the solution for $t=\tau=0.01$. The iteration process is stopped when $\left\|r_{k}\right\|_{\mathcal{M}} /\left\|r_{0}\right\|_{\mathcal{M}} \leq 1 e-8$, and we denote the relative error by $\operatorname{err}=\left\|u-u^{*}\right\|_{l^{2}(\Omega)} /\left\|u^{*}\right\|_{l^{2}(\Omega)}$.

Comparison between ASR and Linearly Implicit Euler methods. We start with a comparison between our ASR method (3.9) and the Linearly Implicit Euler method, consisting in applying to the original nonlinear problem (2.2) a single Newton step, see e.g. Deuflhard [14], Lang [18], and solve the resulting linear system by GMRES with the Additive Schwarz preconditioner $\mathcal{M}$ defined in (3.7). This method has the advantage of requiring only the solution of a linear problem per time step, but it requires the computation of the Jacobian of the nonlinear operator. We remark that the Jacobian is not needed in our ASR algorithm and it might even be practically uncomputable for some nonlinear problems, such as the monodomain and bidomain systems coupled with realistic ionic models; see Munteanu [21]. In our notations, the Linearly Implicit Euler method for the nonlinear system (3.1) $B\left(u^{m}\right)=\hat{g}$ at time step $t_{m}$ becomes:

solve the Jacobian system: $\quad J_{B}^{m} s^{m}=\hat{g}-B\left(u^{m}\right)$

by GMRES with the Additive Schwarz preconditioner $\mathcal{M}$ in (3.7), update the solution: $\quad u^{m+1}=u^{m}+s^{m}$,

where $J_{B}^{m}$ is the Jacobian of the nonlinear operator $B$ at $u^{m}$. Given our assumption (3.2) on the structure of $B$, this Jacobian matrix also can be written in terms of the nonlinear form $b(u, v)$ and the mass matrix $M$ as $J_{B}^{m}=M+\tau J_{b}^{m}$, with $J_{b}^{m}$ equal to the Jacobian of $b$ at $u^{m}$.

Table 6.1 compares the two algorithms by reporting their iteration counts (iter) and MATLAB cpu times (cpu) on a PC (Acer Aspire 3680) in a scaled speed-up test, where the subdomain size $H / h=4$ is kept fixed and the number of subdomains (hence nodes) is increased 
TABLE 6.2

ASR scaled speed-up test with fixed subdomain size $H / h=4$, small overlap size $\delta=h$, and increasing number of subdomains $N$ (and nodes): ASR with fixed $\lambda=0.4$ and random RHS (second and third columns); ASR with step-lenght strategy for $\lambda$ and exact solution (fourth and fifth columns). iter $=$ iteration counts, err $=$ relative errors with exact solution.

\begin{tabular}{c|c|c|cc|cc} 
& \multicolumn{2}{|c|}{$\begin{array}{c}\text { ASR with } \lambda=0.4 \\
\text { random RHS }\end{array}$} & \multicolumn{3}{|c}{ ASR with step-lenght strategy for $\lambda$} \\
& one-level & two-levels & \multicolumn{2}{|c}{ exact solution RHS } \\
one-level & \multicolumn{2}{c}{ two-levels } \\
N & iter & iter & iter & err & iter & err \\
\hline $2 \times 2$ & 40 & 42 & 25 & $6.76 \mathrm{e}-3$ & 24 & $6.76 \mathrm{e}-3$ \\
$4 \times 4$ & 70 & 38 & 37 & $1.67 \mathrm{e}-3$ & 25 & $1.67 \mathrm{e}-3$ \\
$6 \times 6$ & 122 & 39 & 69 & $7.44 \mathrm{e}-4$ & 24 & $7.44 \mathrm{e}-4$ \\
$8 \times 8$ & 196 & 40 & 117 & $4.18 \mathrm{e}-4$ & 24 & $4.18 \mathrm{e}-4$ \\
$10 \times 10$ & 291 & 42 & 157 & $2.67 \mathrm{e}-4$ & 27 & $2.67 \mathrm{e}-4$ \\
$12 \times 12$ & 407 & 41 & 223 & $1.86 \mathrm{e}-4$ & 22 & $1.86 \mathrm{e}-4$ \\
$14 \times 14$ & 544 & 42 & - & - & 25 & $1.36 \mathrm{e}-4$ \\
$16 \times 16$ & 703 & 43 & - & - & 23 & $1.04 \mathrm{e}-4$ \\
$18 \times 18$ & 882 & 43 & - & - & 24 & $8.26 \mathrm{e}-5$
\end{tabular}

from $2 \times 2$ to $18 \times 18$. For both algorithms the right-hand side is chosen so that the exact solution is $u^{*}$ given above and we can determine the relative errors (err) reported in the last column of the table, that decrease proportionally to the mesh refinement (i.e., increasing subdomains) as expected. In agreement with the theory (see Remark 5.3), in the one-level case the number of iterations of both algorithms increases (much less for Linearly Implicit Euler, column 5), while in the two-level case the iteration counts remain bounded and both algorithms are scalable (with a better upper bound of 16 for Linearly Implicit Euler, column 6 , than 36 for ASR, column 3). The cpu times behave accordingly, i.e., they increase strongly for the one-level algorithms and show a more moderate increase for the two-level algorithms. The most relevant comparison between the two-level algorithms shows that the ASR cpu times are initially slightly larger than the Linearly Implicit Euler cpu times, but as the problem size increases (for $N \geq 14 \times 14$ ) the ASR times equal and then definitely improve over the Lineary Implicit Euler times ( 27.08 v. $37.42 \mathrm{sec}$. for $N=16 \times 16$ and 43.18 v. 76.17 sec. for $N=18 \times 18$ ). These results indicate that ASR can be asymptotically more efficient than Linearly Implicit Euler as the problem size and number of subdomains increase. We remark that this is only a partial indication because of the serial implementation of the two algorithms, where the subdomain problems of the Additive Schwarz preconditioners are solved sequentially; it would be much more significant to compare the parallel cpu times for the two algorithms on modern distributed computing architectures (which is beyond the scope of this paper).

ASR scalability with random RHS and $\lambda$ step-lenght strategy. Table 6.2 reports the results of a scaled speed-up test analogous to Table 6.1, but focuses only on the ASR algorithm with minimal overlap $\delta=h$. In the left part of the table (columns 2 and 3), the parameter $\lambda$ is again fixed at 0.4 but the RHS is randomly distributed. In the right part of the table (column 4 and 5), $\lambda$ is chosen by the step-length strategy of [26] (columns 4 and 5 ) and the RHS is again the one associated to the exact solution $u^{*}$ given above. The results confirm that in the one-level case the number of ASR iterations (iter) increases, while in the two-level case this number remains bounded and the ASR algorithm is scalable. The steplength strategy for the selection of $\lambda$ yields better iteration counts (around 22 - 27 iterations) than the fixed $\lambda=0.4$ selection (around 36 iterations), but at the expense of a much larger cpu time (not shown). The results with a random right-hand side show the same scalability of the two-level ASR algorithm, only with slightly larger iteration counts (now with an upper bound of 43 iterations). 
TABLE 6.3

ASR standard speed-up test with fixed mesh size $h=1 / 48$, small overlap size $\delta=h$, and increasing number of subdomains $N$ (of decreasing size $H / h$ ). ASR with fixed $\lambda=0.4$ (second and third columns); ASR with step-lenght strategy for $\lambda$ (fourth and fifth columns). iter $=$ iteration counts, err $=$ relative errors with exact solution.

\begin{tabular}{c|cc|cc|cc|cc} 
& \multicolumn{4}{|c|}{$\lambda=0.4$} & \multicolumn{3}{c}{$\lambda$ : step-length strategy } \\
& \multicolumn{2}{|c|}{ one-level } & \multicolumn{2}{c|}{ two-levels } & \multicolumn{2}{|c}{ one-level } & \multicolumn{2}{c}{ two-levels } \\
$\mathrm{N}$ & iter & err & iter & err & iter & err & iter & err \\
\hline $2 \times 2$ & 155 & $1.74 \mathrm{e}-4$ & 70 & $1.74 \mathrm{e}-4$ & 86 & $1.74 \mathrm{e}-4$ & 43 & $1.74 \mathrm{e}-4$ \\
$4 \times 4$ & 192 & $1.74 \mathrm{e}-4$ & 58 & $1.74 \mathrm{e}-4$ & 114 & $1.74 \mathrm{e}-4$ & 32 & $1.74 \mathrm{e}-4$ \\
$6 \times 6$ & 245 & $1.74 \mathrm{e}-4$ & 47 & $1.74 \mathrm{e}-4$ & 149 & $1.74 \mathrm{e}-4$ & 27 & $1.74 \mathrm{e}-4$ \\
$8 \times 8$ & 301 & $1.74 \mathrm{e}-4$ & 41 & $1.74 \mathrm{e}-4$ & - & - & 23 & $1.74 \mathrm{e}-4$
\end{tabular}

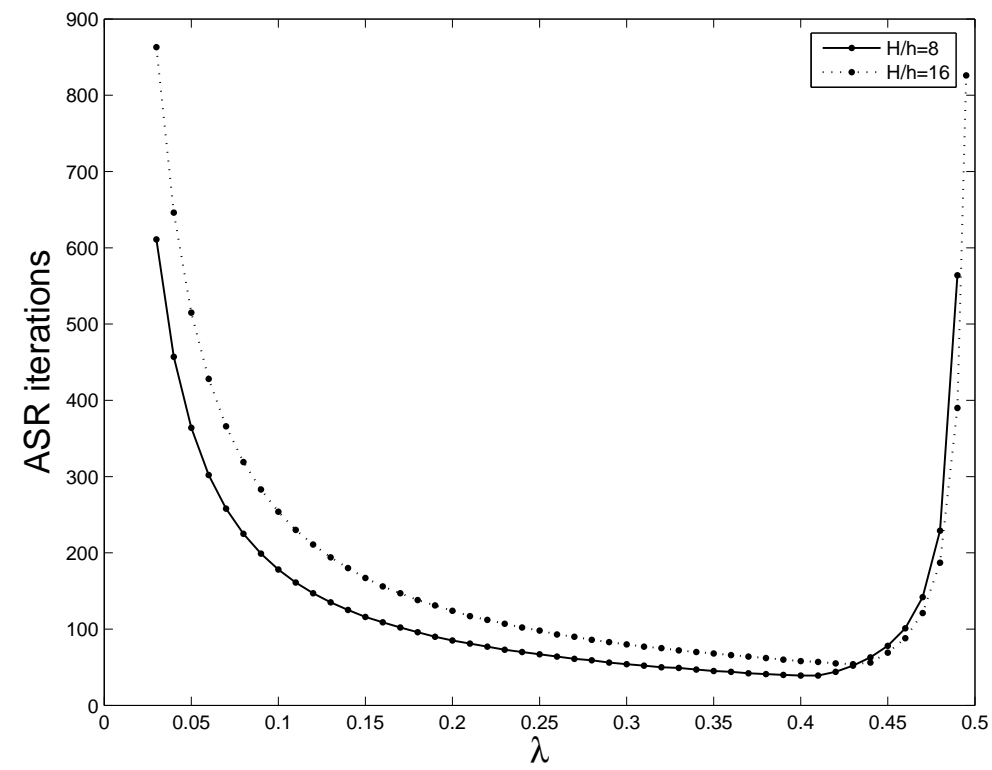

FIG. 6.1. ASR iterations counts as a function of the parameter $\lambda ; N=2 \times 2$, overlap size $\delta=h$.

ASR standard speed-up. We study the ASR performance in a standard speed-up test where the global problem size is fixed $(h=1 / 48)$ and the number of subdomains is increased from $2 \times 2$ to $8 \times 8$, hence decreasing the ratio $H / h$. The same quantities (iter and err) of Table 6.2 are reported in Table 6.3. As predicted by the theory, only in the two-level case, the ASR iteration counts improve as the subdomain size $H$ decreases, since the term $\tau /(H h)$ dominates the one-level bound in Remark 5.3b).

ASR dependence on $\lambda$. Figure 6.1 confirms the theoretical prediction of Theorem 4.7, showing the ASR iteration counts as a function of the parameter $\lambda$ for $N=2 \times 2$ subdomains, overlap $\delta=h$, and two mesh sizes $h=1 / 8$ (continuous line), $h=1 / 16$ (dashed line). The explicit formula of Theorem 4.7 shows that the parabola $P(\lambda)$ attains its minimum inside a right interval of 0 and tends to 1 at its endpoints; correspondingly, the ASR convergence rate attains a minimum inside an interval $(0, \alpha), \alpha>0$ and degenerates at the interval endpoints.

ASR dependence on $\delta$. Table 6.4 shows that the ASR iteration counts improve with increasing overlap size $\delta$, for both the one- and two-level ASR algorithms, in agreement with Lemma 5.1. In the two-level case, the improvement becomes irrelevant for overlap sizes 
TABLE 6.4

Effect of increasing the overlap size $\delta$ in one-and two-level ASR methods for fixed mesh size $h=1 / 48$, number of subdomains $N=2 \times 2, \lambda=0.4$ iter $=$ iteration counts, err $=$ relative errors with exact solution.

\begin{tabular}{c|cc|cc} 
overlap size & \multicolumn{2}{|c|}{ one-level } & \multicolumn{2}{c}{ two-levels } \\
$\delta$ & iter & err & iter & err \\
\hline $\mathrm{h}$ & 155 & $1.74 \mathrm{e}-4$ & 70 & $1.74 \mathrm{e}-4$ \\
$2 \mathrm{~h}$ & 82 & $1.74 \mathrm{e}-4$ & 46 & $1.74 \mathrm{e}-4$ \\
$3 \mathrm{~h}$ & 59 & $1.74 \mathrm{e}-4$ & 37 & $1.74 \mathrm{e}-4$ \\
$4 \mathrm{~h}$ & 49 & $1.74 \mathrm{e}-4$ & 37 & $1.74 \mathrm{e}-4$
\end{tabular}

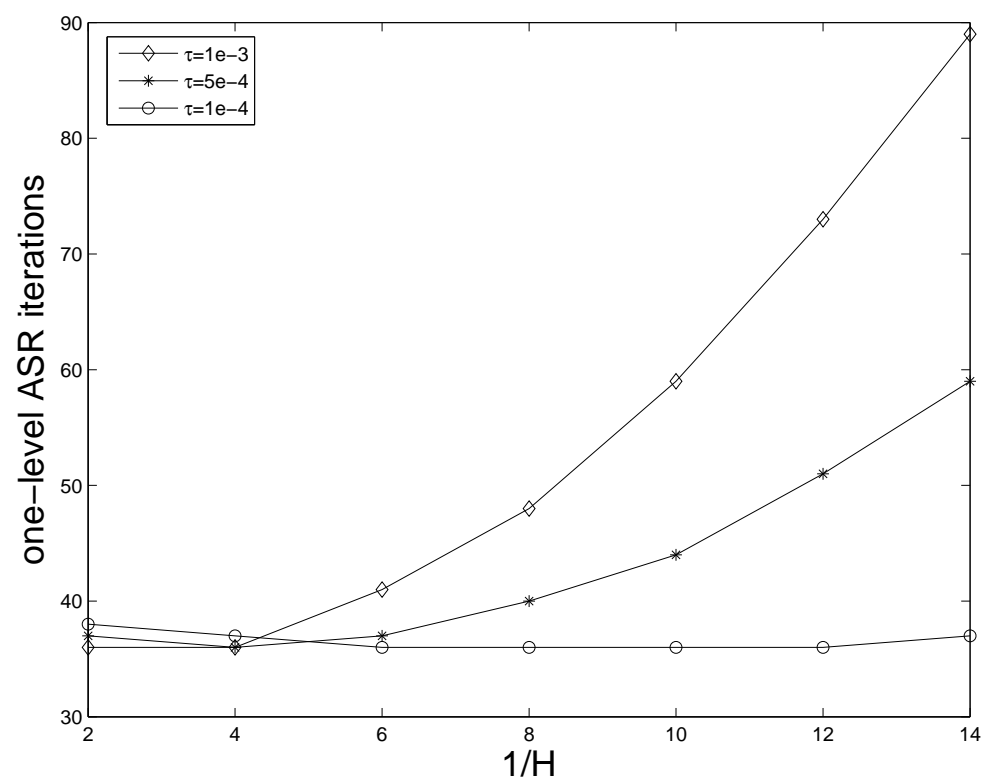

FIG. 6.2. One-level ASR iterations counts for increasing number of subdomains $1 / H$ and different time-step sizes $\tau$, fixed ratio $H / h=4, \delta=h$.

larger than $3 h$.

ASR scalability dependence on $\tau$. Figure 6.2 reports a different validation of the onelevel bound by reducing the time-step size $\tau$ and performing a scaled speed-up test as in Table $6.2(H / h$ fixed and small overlap $\delta=h)$. While we already know that for a given value of $\tau$ the one-level ASR algorithm is not scalable (Remark 5.3 and Table 6.2), we nevertheless expect a reduction of $\tau$ to give bounded iteration counts up to a critical number of subdomains $N_{\tau}$ ("temporary" scalability) since the first term dominates the maximum in Remark 5.3a), while for $N>N_{\tau}$ we expect increasing iteration counts since the second term dominates the maximum in Remark 5.3a). The results in Figure 6.2 show that this is indeed the case: for $\tau=1 e-3, N_{\tau} \sim 4 \times 4$, for $\tau=5 e-4, N_{\tau} \sim 6 \times 6$ and for $\tau=1 e-4, N_{\tau} \sim 12 \times 12$.

ASR performance for elliptic coefficients with jump discontinuities across subdomains. Finally, Figures 6.3 and 6.4 report the ASR results when the coefficients $\sigma_{i}$ of the linear elliptic operator are piecewise constant and present jump discontinuities across subdomain boundaries. We considered a decomposition of $\Omega$ into $7 \times 7$ subdomains with $H / h=4, \delta=h$; we chose a larger tolerance $t o l=1 e-4$ in the stopping criterion in order to test the convergence (or lack thereof) of the unpreconditioned algorithm, up to a maximum 
ETNA

Kent State University

http://etna.math.kent.edu

OVERLAPPING ASR FOR NONLINEAR PARABOLIC PROBLEMS

\begin{tabular}{|c|c|c|c|c|c|c|}
\hline 1 & 1 & 1 & 1 & 1 & 1 & 1 \\
\hline 1 & 1 & 1 & 1 & 1 & 1 & 1 \\
\hline 1 & 1 & $\sigma_{i}$ & $\sigma_{i}$ & $\sigma_{i}$ & 1 & 1 \\
\hline 1 & 1 & $\sigma_{i}$ & $\sigma_{i}$ & $\sigma_{i}$ & 1 & 1 \\
\hline 1 & 1 & $\sigma_{i}$ & $\sigma_{i}$ & $\sigma_{i}$ & 1 & 1 \\
\hline 1 & 1 & 1 & 1 & 1 & 1 & 1 \\
\hline 1 & 1 & 1 & 1 & 1 & 1 & 1 \\
\hline
\end{tabular}

\begin{tabular}{l|c|c|c}
$\sigma_{i}$ & $\begin{array}{c}\text { one-level } \\
\text { iter }\end{array}$ & $\begin{array}{c}\text { two-levels } \\
\text { iter }\end{array}$ & $\begin{array}{c}\text { no prec } \\
\text { iter }\end{array}$ \\
\hline $10^{-2}$ & 63 & 17 & - \\
$10^{-1}$ & 66 & 17 & - \\
1 & 77 & 17 & 29818 \\
10 & 76 & 17 & - \\
$10^{2}$ & 72 & 17 & -
\end{tabular}

FIG. 6.3. Iteration counts of one- and two-level ASR method with $\lambda=0.4$ for a test problem with discontinuous coefficient $\sigma_{i}$ in the linear elliptic operator; the domain is decomposed into $N=7 \times 7$ subdomains (left panel, with values of $\sigma_{i}$ indicated), $H / h=4, t o l=1 e-4$

\begin{tabular}{|c|c|c|c|c|c|c|}
\hline 0 & -3 & 1 & 0 & -1 & 2 & 0 \\
\hline-2 & 2 & 0 & 1 & -3 & 3 & 0 \\
\hline 1 & 0 & -2 & 2 & 3 & 2 & 1 \\
\hline 2 & -3 & 2 & 0 & 2 & 1 & -3 \\
\hline 3 & 0 & 1 & 2 & 0 & 1 & 3 \\
\hline-3 & 1 & 0 & 3 & -3 & 3 & 0 \\
\hline 0 & -1 & 1 & 2 & -2 & 3 & -3 \\
\hline
\end{tabular}

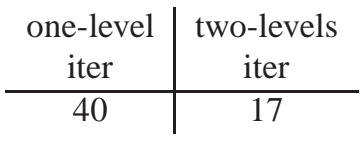

FIG. 6.4. Iteration counts of one- and two-level ASR method with $\lambda=0.4$ for a test problem with random discontinuous coefficient $\sigma_{i}$ in the linear elliptic operator; the domain is decomposed into $N=7 \times 7$ subdomains (left panel), with indicated the values of the exponents $\alpha_{i}$ in the coefficients $\sigma_{i}=10^{\alpha_{i}}, H / h=4$, tol $=1 e-4$

number of iterations maxit $=3 \cdot 10^{4}$. We first considered a configuration where inside the $3 \times 3$ central subdomains $\Omega_{i}$ (see Figure 6.3, left) the elliptic coefficients $\sigma_{i}$ have varying values ranging from $1 e-2$ to $1 e+2$, while they are equal to 1 in the other surrounding subdomains. The table in Figure 6.3, right, show that the two-level ASR method is unaffected by the size of the discontinuity, the one-level is almost unaffected but with iteration counts more than four times larger, and the unpreconditioned Richardson iteration essentially does not converge within maxit iterations. We also considered a second configuration where the coefficients $\sigma_{i}=10^{\alpha_{i}}$ have random exponents $\alpha_{i}$ given in Figure 6.4, left, with a variation of six orders of magnitude. The two-level ASR iteration counts are the same as before (17), the one-level iteration counts are a bit better (40) and the unpreconditioned method again does not converge.

\section{REFERENCES}

[1] H.-B. An, On convergence of the Additive Schwarz Preconditioned Inexact Newton Method, SIAM J. Numer. Anal., 43 (2005), pp. 1850-1871.

[2] L. BADEA, Convergence rate of Schwarz multilevel method for the constrained minimization of nonquadratic functionals, SIAM J. Numer. Anal., 44 (2006), pp 449-477.

[3] L. BADEA, X.-C. TAI, AND J. WANG, Convergence rate analysis of a multiplicative Schwarz method for variational inequalities, SIAM J. Numer. Anal., 41 (2003), pp 1052-1073.

[4] I. BoglaEv, Monotone iterative algorithms for a nonlinear singularly perturbed parabolic problem, J. Comput. Appl. Math., 172 (2004), pp 313-335.

[5] D. BRAESS, Finite Elements. Theory, Fast Solvers, and Applications in Solid Mechanics, Cambridge University Press, Cambridge, 2001.

[6] J. H. Bramble And J. Xu, Some estimates for a weighted L $L^{2}$ projection, Math. Comp., 56 (1991), pp. $463-$ 476

[7] X.-C. CAI, Additive Schwarz algorithms for parabolic convection-diffusion equations, Numer. Math., 60 (1991), pp. 41-61.

[8] X-C. CAI, Multiplicative Schwarz methods for parabolic problems, SIAM J. Sci. Comput., 15 (1994), 
pp. 587-603.

[9] X-C. CAI AND M. DRYJA, Domain decomposition methods for monotone nonlinear elliptic problems, in Seventh International Conference on Domain Decomposition Methods in Scientific and Engineering Computing, D. E. Keyes and J. Xu, eds., Contemp. Math., Vol. 180, American Mathematical Society, Providence, RI, 1994, pp. 21-27.

[10] X-C. Cai, W. D. Gropp, D. E. Keyes, R. G. Melvin, and D. P.Young, Parallel Newton-KrylovSchwarz algorithms for the transonic full potential equation, SIAM. J. Sci. Comput., 19 (1998), pp. 246265.

[11] X-C. Cai, W. D. Gropp, D. E. Keyes, And M. D. Tidriri, Newton-Krylov-Schwarz methods in CFD, in Proceedings of the International Workshop on Numerical Methods for the Navier-Stokes Equations, Vieweg, Braunschweig, 1995, pp. 183-200.

[12] X-C. CAI AND D. E. KeYES, Nonlinearly preconditioned inexact Newton algorithms, SIAM. J. Sci. Comput., 24 (2002), pp. 183-200.

[13] P. COLLI FRANZONE AND L. F. PAVARINO. A parallel solver for reaction-diffusion systems in computational electrocardiology, Math. Models Methods Appl. Sci., 14 (2004), pp. 883-911.

[14] P. DEUfLHARD, Uniqueness theorems for stiff ODE initial value problems, in Numerical Analysis 1989, D. F. Griffiths and G. A. Watson, eds., Pitman Research Notes in Mathematics Series 228, Longman, 1990, pp. 74-88.

[15] M. DRYjA AND W. HaCkBUSH, On the nonlinear domain decomposition method, BIT, 37 (1997), pp. 296311.

[16] M. DRYja And O. B. Widlund, Domain decomposition algorithms with small overlap, SIAM J. Sci. Comput., 15 (1994), pp. 604-620.

[17] F-N. HWANG AND X-C. CAI, A parallel nonlinear additive Schwarz preconditioned inexact Newton algorithm for incompressible Navier-Stokes equations, J. Comput. Phys., 204 (2005), pp. 666-691.

[18] J. LANG, Adaptive Multilevel Solution of Nonlinear Parabolic PDE Systems. Theory, Algorithm, and Applications. Lect. Notes Comput. Sci. Eng., Vol. 16, Springer, Berlin, 2000.

[19] S-H. LuI, On linear monotone iteration and Schwarz methods for nonlinear elliptic PDEs, Numer. Math., 93 (2002), pp. 109-129.

[20] S-H. LUI, On monotone iteration and Schwarz methods for nonlinear parabolic PDEs, J. Comput. Appl. Math., 161 (2003), pp. 449-468.

[21] M. MunTEANU, Overlapping additive Schwarz methods for nonlinear parabolic reaction-diffusion problems, Ph.D. Thesis, Dept. of Mathematics, University of Milano, 2008.

[22] M. MunteAnU AND L. F. PAVARINO, Implicit parallel solvers in computational electrocardiology. in Applied Analysis and Differential Equations, O. Carja and I. I. Vrabie, eds., World Scientific, 2007, pp. 255266.

[23] M. Munteanu And L. F. Pavarino, An Overlapping Additive Schwarz-Richardson Method for Monotone Nonlinear Parabolic Problems, in Domain Decomposition Methods in Science and Engineering XVII, U. Langer et al., eds., Lect. Notes Comput. Sci. Eng., Vol. 60, Springer, Berlin, 2008, pp. 599-606.

[24] M. MUNTEANU AND L. F. PAVARINO, Decoupled Schwarz algorithms for implicit discretization of nonlinear monodomain and bidomain systems, Math. Models Methods Appl. Sci., 19 (2009), to appear.

[25] M. MuRILlo AND X-C. CAI, A fully implicit parallel algorithm for simulating the non-linear electrical activity of the heart, Numer. Linear Algebra Appl., 11 (2004), pp. 261-277.

[26] J. Nocedal and S. J. Wright, Numerical Optimization, Springer, New York, 1999.

[27] S. Ovtchinnikov AND X-C. CAI, One-level Newton-Krylov-Schwarz algorithm for unsteady non-linear radiation diffusion problem, Numer. Linear Algebra Appl., 10 (2004), pp. 867-881.

[28] L. F. PAVARINO AND S. SCACCHI, Multilevel additive Schwarz preconditioners for the bidomain reactiondiffusion system, SIAM J. Sci. Comput., 31 (2008), pp. 420-443.

[29] B. F. Smith, P. E. BJøRstad, And W. D. Gropp, Domain Decomposition. Parallel Multilevel Methods for Elliptic Partial Differential Equations, Cambridge University Press, Cambridge, 1996.

[30] J. Sundnes, G. T. Lines, X. Cai, B. F. Nielsen, K.-A. Mardal, and A. Tveito, Computing the Electrical Activity of the Heart, Springer, Berlin, 2006.

[31] X-C. TAI AND M. EsPEDAL, Rate of convergence of some space decomposition methods for linear and nonlinear problems, SIAM J. Numer. Anal., 35 (1998), pp. 1558-1570.

[32] X-C. TAI AND M. EsPEDAL, Applications of a space decomposition method to linear and nonlinear elliptic problems, Numer. Methods Partial Differential Equations, 14 (1998), pp. 717-73.

[33] A. Toselli And O. Widlund, Domain Decomposition Methods-Algorithms and Theory, Springer, Berlin, 2005

[34] J. XU AND X-C. CAI, A preconditioned GMRES method for nonsymmetric or indefinite problems, Math. Comp., 59 (1992), pp. 311-319.

[35] A. ŽENIŠEK, Nonlinear elliptic and evolution problems and their finite element approximations, Academic Press, London, 1990.

[36] X. Zhang, Multilevel Schwarz methods, Numer. Math., 63 (1992), pp. 521-539. 